\title{
Numerical Simulation Analysis of Ultrafine Powder Centrifugal Classifier Bizhong XIA ${ }^{1,}$, Yiwei $\mathrm{CHEN}^{1,}$, , Bo $\mathrm{CHEN}^{2}$ \\ ${ }^{1}$ Graduate School at Shenzhen, Tsinghua University, Shenzhen, 518055, China \\ ${ }^{2}$ ShenZhen KeLi Force Nano Engineering Equipment Co. Ltd, Shenzhen, 518408, China \\ aemail: xiabz@sz.tsinghua.edu.cn, bemail: chenyiwei1990@126.com
}

Key words: Numerical Simulation; Centrifugal Classification; Cut Size

\begin{abstract}
Numerical simulation of liquid-solid two-phase flow was used to analyze the flow of the fluid in the classification chamber of ultrafine powder centrifugal classifier. Under the condition of the steady state, in order to study the influence of different blade angles on the classification performance, Eulerian multiphase flow model, RNG k- $\varepsilon$ turbulence model and multiple reference frame (MRF) method were used to analyze the velocity distribution of the fluid in the classification chamber. On this basis, under the condition of the unsteady state, in order to study the influence of different classification wheel rotate speed on the cut size, the discrete phase model (DPM) was used to analyze the rule of particle track in the classification chamber. The results indicate that: when the blade angle is $15 \sim 30^{\circ}$, the classification performance is the best; When the blade angle is $30^{\circ}$, the cut size is close to $1 \mu \mathrm{m}$ when the classification wheel speed is 1000rpm; The cut size is close to 0.5 $\mu \mathrm{m}$ when the classification wheel speed is 2000rpm; The cut size is smaller than $0.5 \mu \mathrm{m}$ when the classification wheel speed is 3000rpm. The simulation results well agree with the experimental results.
\end{abstract}

\section{Introduction}

With the development of modern industry, it is becoming stricter to the requirement of powder. How to produce ultrafine powder with a narrow particle size distribution has become a problem of powder preparation [1]. Under this condition, powder classification technology has been a subject of great interest over the past decades due to the potential applications in various industries, including industrial pharmacy, mining and chemical engineering. As one of the most important indicators to evaluate the classification effect, the cut size of classifiers draws considerable concern. A number of methods have been developed to evaluate the classification efficiency. One of the most recognized methods is numerical simulation method, which analyze the velocity distribution of the fluid in the classification chamber. Many scholars have done a lot of research in this field. Such as, Du et al. [2] concluded that rotate speed, blade spacing and blade angle have great influence on the particle trajectory by using single particle dynamics model to simulate the particle trajectory. Gao et al. [3] studied the relationship between turbine speed and cut size by using numerical simulation method on turbo air classifier. S. M. Mousavian and A. F. Najafi [4] investigated the influence of geometry on separation efficiency in a hydro cyclone by using a numerical study of gas-liquid-solid multiphase flow. Robert Johansson and Magnus Evertsson [5] used CFD simulation to improve the understanding of the influence of the geometric design of a centrifugal air classifier on the cut size and the particle size distribution, and the simulation results show that the classification results are affected by air flow velocity, particle shape, particle size, the geometry of the air classifier and the turbulence in the air flow. 


\section{Model Descriptions}

The schematic diagram of the ultrafine powder centrifugal classifier used in the present study is shown in Figure 1. Classification wheel rotates by the driving belt, and it forms centrifugal force field in the classification chamber. The particles are subject to the centrifugal force and the fluid drag force. The coarse particles are mainly affected by the centrifugal force, and move to the boundary. The fine particles are mainly affected by the fluid drag force, and flow out from fine powder outlet. Coarse powder outlet is usually closed. In order to let the coarse particle flow out, need to open coarse powder outlet for a period of time while classifier working.

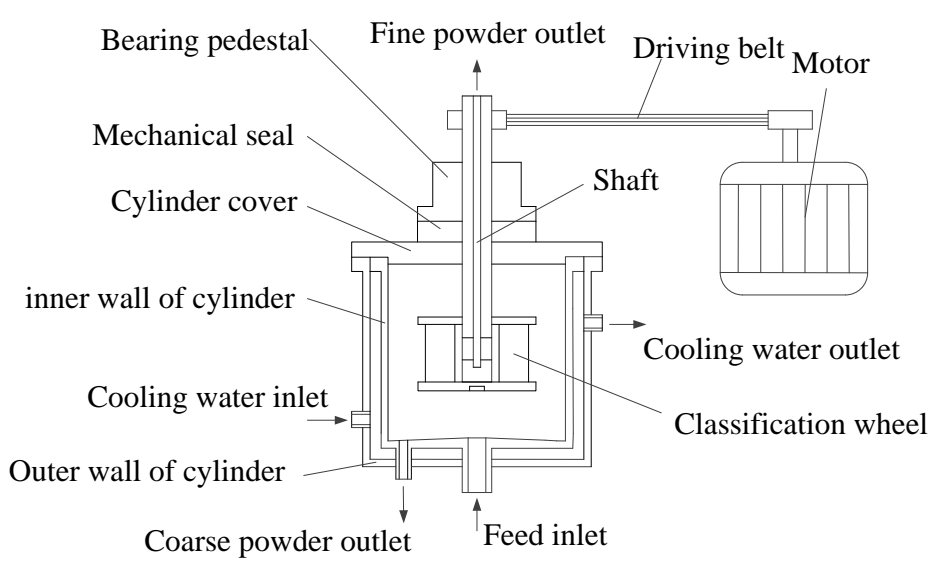

Fig. 1. Schematic diagram of the ultrafine powder centrifugal classifier

The flow media is composed by water and particles while ultrafine powder centrifugal classifier working. It belongs to the category of liquid-solid two-phase flow, which regards the fluid as continuous medium and the particles as pseudo-fluid. Based on the Eulerian-Eulerian liquid-solid two-phase flow model, governing equations include mass conservation equation (continuity equation) and momentum conservation equation (N-S equation) while heat exchange and mass exchange ignored between phase to phase [6].

The mass conservation equation of fluid phase is written as follows:

$$
\frac{\partial \rho_{f}}{\partial t}+\frac{\partial\left(\rho_{f} u_{f j}\right)}{\partial x_{j}}=0
$$

The momentum conservation equation of fluid phase is written as follows:

$$
\frac{\partial \rho_{f} u_{f i}}{\partial t}+\frac{\partial\left(\rho_{f} u_{f j}{ }^{f} f i\right.}{\partial x_{j}}=-\frac{\partial p}{\partial x_{i}}+\frac{\partial}{\partial x_{j}}\left[\mu_{e}\left(\frac{\partial u_{f i}}{\partial x_{j}}+\frac{\partial u_{f j}}{\partial x_{i}}\right)\right]+\rho_{f} f_{i}+\frac{\rho_{s}}{\tau_{r s}}\left(u_{s i}-u_{i}\right)
$$

The mass conservation equation of solid phase is written as follows:

$$
\frac{\partial \rho_{s}}{\partial t}+\frac{\partial\left(\rho_{s} u_{s j}\right)}{\partial x_{j}}=\frac{\partial}{\partial x_{j}}\left(\frac{v_{s}}{\sigma_{s}} \frac{\partial \rho_{s}}{\partial x_{j}}\right)
$$

The momentum conservation equation of solid phase is written as follows:

$$
\frac{\partial\left(\rho_{s} u_{s i}\right)}{\partial t}+\frac{\partial\left(\rho_{s} u_{s j} u_{s i}\right)}{\partial x_{j}}=\frac{\partial}{\partial x_{j}}\left[\mu_{s}\left(\frac{\partial u_{s i}}{\partial x_{j}}+\frac{\partial u_{s j}}{\partial x_{i}}\right)\right]+\frac{\rho_{s}}{\tau_{r s}}\left(u_{i}-u_{s i}\right)+\frac{\partial}{\partial x_{j}}\left[\frac{v_{s}}{\sigma_{s}}\left(\frac{\partial \rho_{s}}{\partial x_{j}} u_{s i}+\frac{\partial \rho_{s}}{\partial x_{i}} \mu_{s j}\right)\right]+\rho_{s} f_{s i}
$$

In these equations, the subscript $f$ represents fluid phase and the subscript $s$ represents solid phase; $t$ represents time; $\rho$ is the density; $u$ is the velocity; $p$ is the pressure; $\mu_{e}$ is the equivalent viscous coefficient; $\tau_{r s}$ is the relaxation time of particle movement; $\sigma_{s}$ is the turbulent Schmidt number; $v_{s}$ is the turbulent diffusivity coefficient.

The classification chamber is mainly constituted of classification wheel, shaft and cylinder. The geometric model of classification wheel is shown in Figure 2. Pressure-Velocity Coupling was provided by the SIMPLER method. Eulerian multiphase flow model, RNG k- $\varepsilon$ turbulence model 
and multiple reference frame (MRF) method were used. In order to calculate precisely, the non-equilibrium wall function was used for near-wall treatments while the second-order upwind scheme was used for discretization. Heat/mass transfer of particles was ignored when performing calculation [7] [8]. The primary phase was defined as water and the secondary phase was defined as particle $\left(\mathrm{SiO}_{2}\right)$. The powder's density is $2200 \mathrm{~kg} / \mathrm{m}^{3}$. The inlet velocity of two-phase flow was set as $2 \mathrm{~m} / \mathrm{s}$. The outlet was defined as a pressure outlet with $101,325 \mathrm{~Pa}$. The volume fraction of particles was set as 0.2 . The rotary speed of the classification wheel was set as $1000 \mathrm{rpm}$, 2000rpm and 3000rpm respectively.

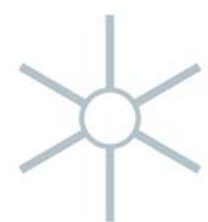

a) $\theta=0^{\circ}$

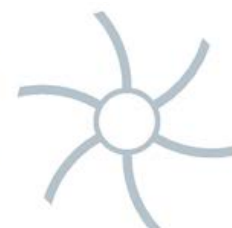

b) $\theta=15^{\circ}$

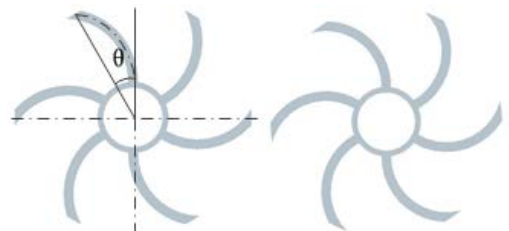

c) $\theta=30^{\circ}$ d) $\theta=45^{\circ}$

Fig. 2 The geometric model of classification wheel

\section{Simulation Results and Discussion}

To study the velocity distribution of axial direction, radial direction and tangential direction, a line named $L$ from $(-90,0,0)$ to $(90,0,0)$ was established. The positive direction of $Y$ axis was set as the positive direction of the axial velocity. The direction from classification wheel to the wall of cylinder was set as the positive direction of the radial velocity. The direction of classification wheel rotating was set as the positive direction of the tangential velocity. Whereas negative direction.

Figure 3 shows the axial velocity distribution of line L. It reveals that: for different blade angles, there are no significant differences of the axial velocity distribution. The axial velocity profiles generally like wavy profiles. The maximum value appears near the center hole of the shaft. There is no large fluctuation in the classification area. Therefore, there is no obvious axial eddy-current, which is conductive to forming stable classification flow field.

Figure 4 shows the radial velocity distribution of line L. There are no significant differences between $\theta=0^{\circ}, \theta=15^{\circ}$ and $\theta=30^{\circ}$. However, the radial velocity profile of $\theta=45^{\circ}$ varies a lot compared the above. When $\theta=45^{\circ}$, the values of radial velocity are positive on the left side of $X=0$, which is not conductive to the fine powder's outflow.

Figure 5 shows the tangential velocity distribution of line L. The particles were subject to the centrifugal force and the fluid drag force while the classifier working. Because the tangential velocity has a great influence on the values of the centrifugal force, it is very necessary to analyze the tangential velocity distribution in order to improve the classification efficiency. As shown in figure 8 , for different blade angles, the maximum value appears at the tip of the blades. When $\theta=45^{\circ}$, the tangential velocity near the tip of the blades changes rapidly. It would cause the centrifugal force changing rapidly, which would affect the stability of the classification flow field.

On the basis of the above discussion, when $\theta=15 \sim 30^{\circ}$, compared with $\theta=0^{\circ}, \theta=45^{\circ}$, the classification performance is the best. On the one hand, velocity contours distribute relatively densely, which is conductive to particles dispersing and would prevent particles conglomerating; on the other hand, there are not great gradients of velocity at the tip of the blades, which is conductive to form a stable classification flow field. 


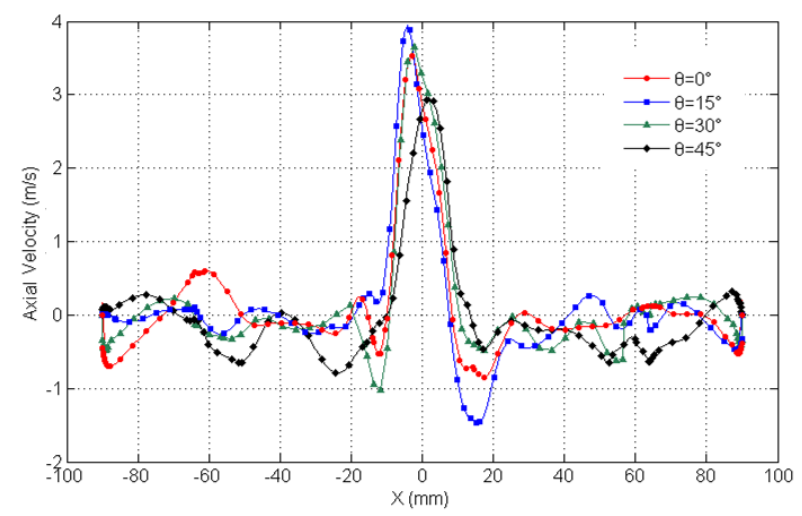

Fig. 3. Axial velocity distribution

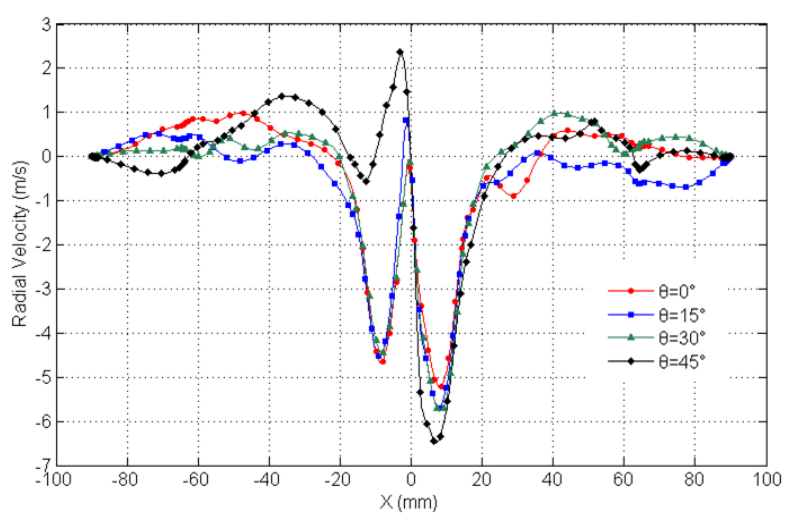

Fig. 4. Radial velocity distribution

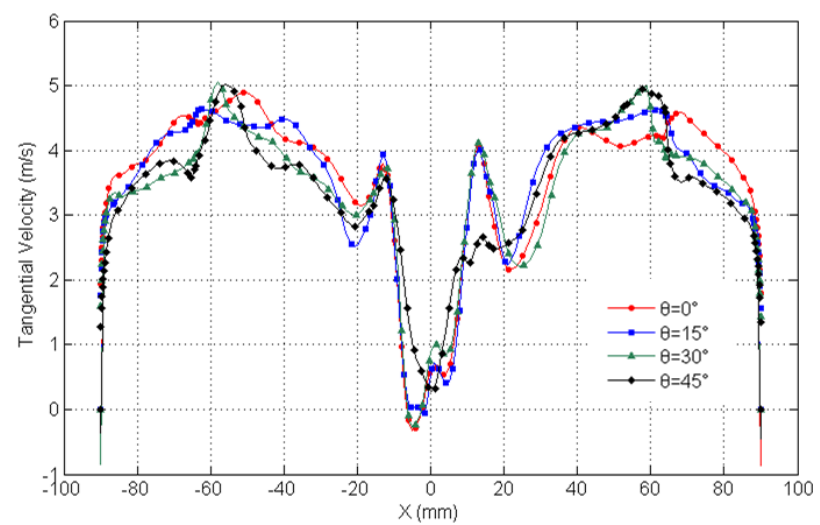

Fig. 5. Tangential velocity distribution

\section{Investigate of Cut Size}

Cut size $d_{50}$ is usually defined as the diameter of a spherical particle for which the inertial centrifugal force offsets the fluid drag force, keeping the same opportunity to enter the coarse powder outlet and the fine powder outlet [9]. According to data, a number of methods have been developed to calculate the cut size in theory [10] [11]. However, all of these methods cannot predict the cut size precisely. In this paper, a new strategy was introduced to determine the cut size using FLUENT discrete phase model (DPM) coupled with experiments. By using FLUENT DPM method, the particle trajectory and velocity could be calculated by analyze the force situation of each particle after calculating the continuous flow field. The cut size was simulated by injecting different size particles and tracking their movement [12]. The density of the material is maintained constant at $2200 \mathrm{~kg} / \mathrm{m}^{3}$. Since the volume fraction of the particle was set as 0.1 , there is no need for momentum coupling between the solid phase and water phase.

Figure 6 shows the particle trajectories of different diameters while the rotary speed of the classification wheel $\mathrm{N}=1000 \mathrm{rpm}$. Different colors of the particle trajectories represent the residence time. When the particle diameter $\mathrm{D}=0.5 \mu \mathrm{m}$, the fluid drag force is greater than the inertial centrifugal force, the particle moves inside and eventually flows out from the fine powder outlet. When $D=1 \mu \mathrm{m}$, the fluid drag force offsets the inertial centrifugal force, the particle rotates in the annular region. When $\mathrm{D}=5 \mu \mathrm{m}$, the fluid drag force is smaller than the inertial centrifugal force, the particle moves outside and eventually flows out from the coarse powder outlet.

Figure 7 shows the particle trajectories of different rotary speeds of the classification wheel while the particle diameter $D=0.5 \mu \mathrm{m}$. The higher the rotary speed of the classification wheel, the greater the inertial centrifugal force. The particle moves inside while $\mathrm{N}=1000 \mathrm{rpm}$, rotates in the annular region while $\mathrm{N}=2000 \mathrm{rpm}$, and moves outside while $\mathrm{N}=3000 \mathrm{rpm}$. 


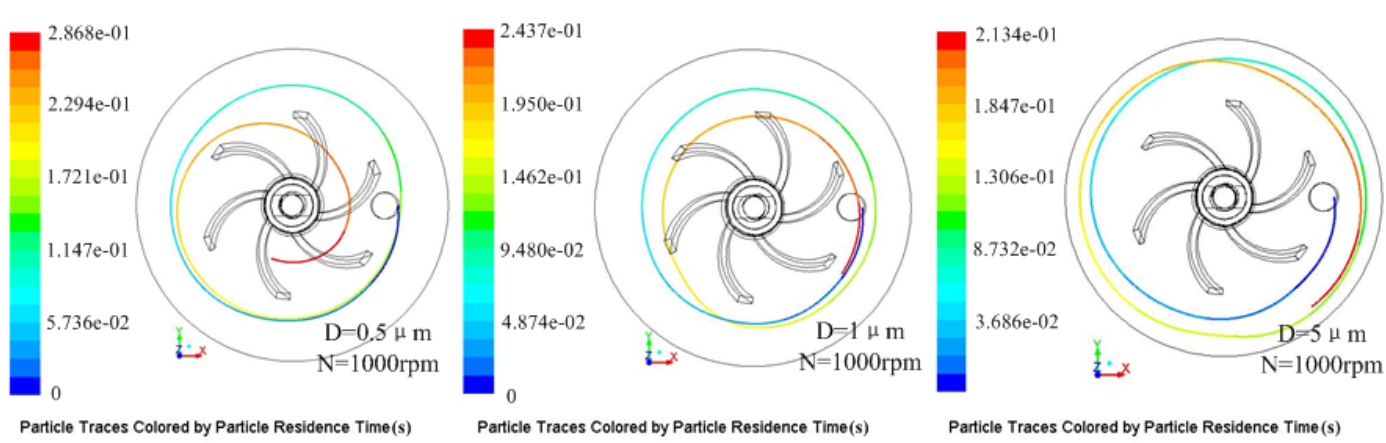

Fig. 6. Particle trajectories when the rotary speed of the classification wheel $\mathrm{N}=1000 \mathrm{rpm}$

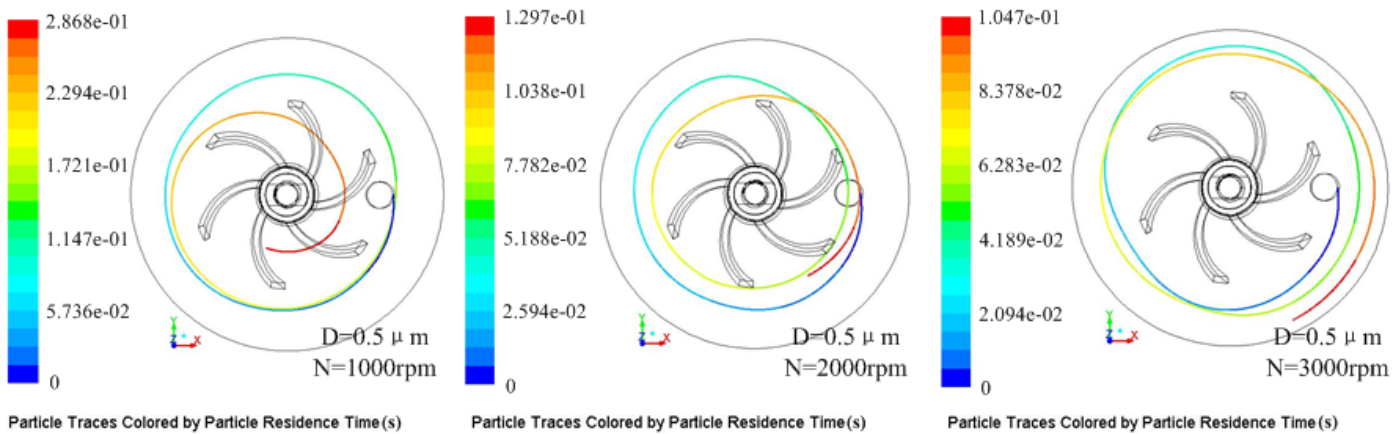

Fig. 7. Particle trajectories of different rotary speeds of the classification wheel

Simulation results show that, when the rotary speed of the classification wheel is 1000rpm, the cut size is close to $1 \mu \mathrm{m}$; when the rotary speed of the classification wheel is $2000 \mathrm{rpm}$, the cut size is close to $0.5 \mu \mathrm{m}$; when the rotary speed of the classification wheel is $3000 \mathrm{rpm}$, the cut size is smaller than $0.5 \mu \mathrm{m}$.

Silicon dioxide powder was selected as experimental material, and its density is $2200 \mathrm{~kg} / \mathrm{m}^{3}$. The experiment was conducted at the rotary speed of the classification wheel of 1000rpm, 2000rpm and $3000 \mathrm{rpm}$, and the inlet velocity of $2 \mathrm{~m} / \mathrm{s}$. It can be seen from table 1 , the simulation results well agree with the experimental data.

Table 1. The comparison of the cut sizes between simulation results and experiment results

\begin{tabular}{lll}
\hline Rotary speed $(\mathrm{N})$ & $\mathrm{d}_{50}$ of simulation results & $\mathrm{d}_{50}$ of experiment results \\
\hline $1000 \mathrm{rpm}$ & $\approx 1 \mu \mathrm{m}$ & $1.27 \mu \mathrm{m}$ \\
$2000 \mathrm{rpm}$ & $\approx 0.5 \mu \mathrm{m}$ & $0.62 \mu \mathrm{m}$ \\
$3000 \mathrm{rpm}$ & $<0.5 \mu \mathrm{m}$ & $0.38 \mu \mathrm{m}$ \\
\hline
\end{tabular}

\section{Conclusions}

In summary, by using the numerical simulation, the present study not only analyzed the influence of different blade angles of the classification wheel on the classification flow field, but also the relationship between the cut size and the rotary speed of the classification wheel. Furthermore, through the experiments, the simulation results about the cut size were verified. Several conclusions can be obtained as follows:

(1) By using Eulerian multiphase flow model, RNG k- $-\varepsilon$ turbulence model and multiple reference frame (MRF) method, the influence of different blade angles on the classification performance was studied. When the blade angle is $15-30^{\circ}$, the classification performance is the best and the classification efficiency is highest.

(2) On the basis of the analysis of the liquid-solid two-phase flow, the trajectories of each particle were studied by using discrete phase model. Simulation results shows: when the inlet velocity of two-phase flow is $2 \mathrm{~m} / \mathrm{s}$ and the volume fraction of the particle is set as 0.1 , the cut size is close to 1 
$\mu \mathrm{m}$ when the classification wheel speed is $1000 \mathrm{rpm}$; the cut size is close to $0.5 \mu \mathrm{m}$ when the classification wheel speed is $2000 \mathrm{rpm}$; the cut size is smaller than $0.5 \mu \mathrm{m}$ when the classification wheel speed is 3000rpm.

(3) The material experiments were done with different rotary speeds of the classification wheel, and the simulation results well agree with the experimental results. The simulation method provides a new method to predict the cut size of an ultrafine Powder Centrifugal Classifier, as well as various types of classifier.

\section{References}

[1] K.G.H. Heiskanen. Developments in wet classifiers [J]. Int. J. Miner. Process, 1996, 44(45): 29-41.

[2] Yanchen Du, Shulin Wang. Particle trajectories in vortex classifier rotor [J]. Journal of Chemical Industry and Engineering, 2005, 56(5): 823-828.

[3] Liping Gao, Yuan Yu, Jiaxiang Liu. Study on the cut size of a turbo air classifier [J]. Powder Technology, 2013, 237: 520-528.

[4] S. M. Mousavian, A. F. Najafi. Influence of geometry on separation efficiency in a hydrocyclone [J]. Arch Appl Mech, 2009, 79: 1033-1055.

[5] Robert Johansson, Magnus Evertsson. CFD simulation of a centrifugal air classifier used in the aggregate industry [J]. Minerals Engineering, 2014, 63: 149-156.

[6] Fan Jiang, Peng Huang. Advanced application and example analysis of fluent [M]. Beijing: Tsinghua University press, 2008.

[7] G. R. Kasata, A. R. Khopkarb. CFD simulation of liquid-phase mixing in solid-liquid stirred reactor [J]. Chemical Engineering Science, 2008, 63: 3877-3885.

[8] Hongzhi Yan, Xinming Li, Bo Wu, et al. Numerical analysis of liquid-solid two-phase in horizontal biaxial stirred tank [J]. Journal of Central South University (Science and Technology), 2013, 44(2): 533-539.

[9] Huizhong Liu, Rongtian Zhen. Hydraulic (wet) classification technology of fine particle [J]. Mining \& Metallurgy, 2005, 14(3): 26-29.

[10] Jiaxiang Liu, Jingbo Xia, Tingshu He, Air flow field characteristics analyzing and classification process of the turbo classifier [J]. Journal of the Chinese Ceramic Society, 2003, 31: 485-489.

[11] Tao Ye, Xu. Ning, Zhichu Huang, et al. Experimental investigation on classified particles cut size of turbo air classifier [J]. Mining \& Processing Equipment, 2006, 34: 62-63.

[12] Cong Tong, Shuangyue Li, Xiang Li. Numerical simulation on particles classification trajectory using unsteady tracking [J]. Chemical Industry and Engineering Progress, 2013, 32(9): 2061-2067. 